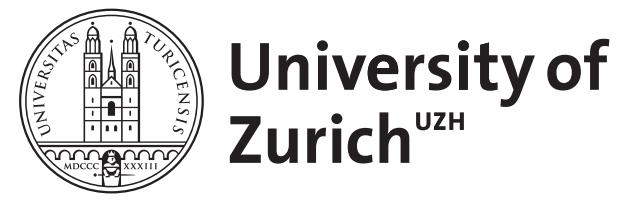
Archive

University of Zurich

University Library

Strickhofstrasse 39

CH-8057 Zurich

www.zora.uzh.ch

Year: 2004

\title{
Maximal regularity for nonautonomous evolution equations
}

\author{
Amann, $\mathrm{H}$
}

\begin{abstract}
We derive sufficient conditions, perturbation theorems in particular, for nonautonomous evolution equations to possess the property of maximal Lp regularity.
\end{abstract}

DOI: https://doi.org/10.1515/ans-2004-0404

Posted at the Zurich Open Repository and Archive, University of Zurich ZORA URL: https://doi.org/10.5167/uzh-21760

Journal Article

Accepted Version

Originally published at:

Amann, H (2004). Maximal regularity for nonautonomous evolution equations. Advanced Nonlinear Studies, 4(4):417-430.

DOI: https://doi.org/10.1515/ans-2004-0404 


\title{
Maximal regularity for nonautonomous evolution equations
}

\author{
H. Amann \\ Institut für Mathematik, \\ Universität Zürich, Winterthurerstr. 190, CH-8057 Zürich, Switzerland \\ e-mail: amann@math.unizh.ch
}

To Antonio Ambrosetti for his $60^{\text {th }}$ birthday

\begin{abstract}
We derive sufficient conditions, perturbation theorems in particular, for nonautonomous evolution equations to possess the property of maximal $L_{p}$ regularity.

1991 Mathematics Subject Classification. 35K90, 47D06.

Key words. Maximal regularity, perturbation theorems, nonautonomous parabolic evolution equations.
\end{abstract}

\section{Introduction}

Let $E_{0}$ and $E_{1}$ be Banach spaces such that $E_{1}$ is continuously and densely embedded in $E_{0}$. Suppose that $J$ is a nontrivial compact subinterval of $\mathbb{R}^{+}$containing zero, and $1<p<\infty$. Then

$$
\mathbb{W}_{p}^{1}\left(J,\left(E_{1}, E_{0}\right)\right):=W_{p}^{1}\left(J, E_{0}\right) \cap L_{p}\left(J, E_{1}\right)
$$

and $\mathbb{W}_{p, 0}^{1}\left(J,\left(E_{1}, E_{0}\right)\right)$ is the closed linear subspace thereof consisting of all $u$ satisfying $u(0)=0$.

Denote by $\mathcal{L}\left(E_{1}, E_{0}\right)$ the Banach space of all bounded linear maps from $E_{1}$ into $E_{0}$, and by $\mathcal{L}$ is $\left(E_{1}, E_{0}\right)$ the set of all isomorphisms therein. Assume that

$$
A \in L_{1}\left(J, \mathcal{L}\left(E_{1}, E_{0}\right)\right) \cap \mathcal{L}\left(\mathbb{W}_{p}^{1}\left(J,\left(E_{1}, E_{0}\right)\right), L_{p}\left(J, E_{0}\right)\right) .
$$

Then $A$ is said to possess (the property of) maximal $L_{p}$ regularity (on $J$ with respect to $\left.\left(E_{1}, E_{0}\right)\right)$ if, given any $f \in L_{p}\left(J, E_{0}\right)$, the initial value problem

$$
\dot{u}+A u=f \quad \text { in } J, \quad u(0)=0
$$


has a unique solution $u$ in $\mathbb{W}_{p}^{1}\left(J,\left(E_{1}, E_{0}\right)\right)$. This is the case iff

$$
\partial+A \in \mathcal{L} \operatorname{is}\left(\mathbb{W}_{p, 0}^{1}\left(J,\left(E_{1}, E_{0}\right)\right), L_{p}\left(J, E_{0}\right)\right),
$$

where $\partial$ is the distributional derivative.

It is known that the assumption of maximal $L_{p}$ regularity puts restrictive conditions on $A$. In fact, in the autonomous case, that is, if $A$ is independent of $t \in J$, the operator $-A$ generates an analytic semigroup on $E_{0}$ if it has the property of maximal $L_{p}$ regularity. Thus maximal regularity is restricted to abstract parabolic evolution equations.

However, maximal regularity is a very useful tool in the study of linear and nonlinear parabolic problems. Indeed, besides of giving optimal results under minimal regularity assumptions on the coefficients of the differential operators (cf. [3]), in the investigation of nonlinear equations it facilitates the application of linearization techniques based on the implicit function theorem (e.g., [11]).

In concrete situations it is no easy task to verify that $A$ possesses the property of maximal $L_{p}$ regularity, even if $A$ is constant. Therefore it is important to have at our disposal general theorems which allow to derive the desired property for large classes of operators if it is already known to hold for certain subclasses. In other words, it is important to have general but simple perturbation theorems. It is the purpose of this paper to derive such theorems.

The first general maximal regularity result seems to be due to De Simon [18] in the autonomous case, that is, if $A$ is constant. He showed that $A$ has maximal $L_{p}$ regularity for every $p>1$ if $-A$ generates an analytic semigroup on the Hilbert space $E_{0}$. The restriction to Hilbert spaces is essential by a recent result of Kalton and Lancien [15] who proved that if $E_{0}$ is not isomorphic to a Hilbert space then there is at least one $A$ not possessing the property of maximal regularity. Consequently, in order to guarantee maximal regularity in the case of Banach spaces one has to impose additional restrictions on the spaces and/or operators. One of the first results of this type is due to Dore and Venni [10]. They showed that $A$ has maximal regularity, provided it possesses bounded imaginary powers and $E_{0}$ is a UMD space. (We refer to [1, Section III.4] for definitions and a detailed exposition of this result.)

More recently, maximal regularity results attracted much attention. In particular, Weis (see [22], [23]) characterized those operators on UMD spaces which possess maximal regularity in terms of $\mathcal{R}$-boundedness. The latter concept - implicitly contained in the work of Bourgain on Fourier multiplier theorems (e.g., [6]) - has been introduced and investigated by Clément and Prüss [7]. (See [8] for a detailed exposition of the theory of $\mathcal{R}$-boundedness, including a proof of Weis' characterization theorem.)

Our main result is Theorem 7.1. It guarantees, in particular, that $A$ possesses the property of maximal $L_{p}$ regularity if this is true for each constant operator $A(\tau)$ with $\tau \in J$, and if $\tau \mapsto A(\tau)$ is continuous. In addition, it is a perturbation theorem which generalizes all comparable known results of this type (see Remarks 7.1). Its proof, as all the other ones in this paper, is very simple. The main idea is to use 
property (1.4) and stability results for bounded invertibility, based on Neumann series.

For applications of our main theorem we refer to [3] and [4]. Further use of it will be made in a forthcoming paper where we study quasilinear parabolic problems in maximal regularity settings.

\section{Preliminaries}

Let $E$ and $F$ be Banach spaces. Recall that $\mathcal{L}$ is $(E, F)$ is open in $\mathcal{L}(E, F)$. More precisely, given $A \in \mathcal{L}$ is $(E, F)$, it follows that $A+B \in \mathcal{L}$ is $(E, F)$ and

$$
\left\|(A+B)^{-1}\right\| \leq 2\left\|A^{-1}\right\| \text { for } B \in \mathcal{L}(E, F) \text { with } 2\|B\| \leq 1 /\left\|A^{-1}\right\| .
$$

Moreover, the inversion

$$
B \mapsto B^{-1}: \mathcal{L} \operatorname{is}(E, F) \rightarrow \mathcal{L} \text { is }(F, E)
$$

is smooth. We set $\mathcal{L}$ aut $(E):=\mathcal{L}$ is $(E, E)$.

A map $R \in \mathcal{L}(E, F)$ is said to be a retraction from $E$ onto $F$ if there exists a continuous right inverse, a coretraction, for $R$.

We write $E \hookrightarrow F$ if $E$ is continuously injected in $F$, that is, $E$ is a linear subspace of $F$ and the natural injection $x \mapsto x$ belongs to $\mathcal{L}(E, F)$. Furthermore, $E \stackrel{d}{\hookrightarrow} F$ means that $E$ is also dense in $F$.

We denote by $\mathcal{H}(F)$ the set of closed and densely defined linear operators $A$ in $F$ such that $-A$ generates a strongly continuous analytic semigroup, $\left\{e^{-t A} ; t \geq 0\right\}$, on $F$, that is, in $\mathcal{L}(F):=\mathcal{L}(F, F)$. If $E \stackrel{d}{\hookrightarrow} F$ then $\mathcal{H}(E, F):=\mathcal{L}(E, F) \cap \mathcal{H}(F)$.

Throughout this paper

$\left.\begin{array}{l}\text { - } E_{0} \text { and } E_{1} \text { are Banach spaces with } E_{1} \stackrel{d}{\hookrightarrow} E_{0} \text { and } \mathcal{H}\left(E_{1}, E_{0}\right) \neq \emptyset ; \\ \text { - } 1<p<\infty \text {. }\end{array}\right\}$

We write $(\cdot, \cdot)_{\theta, r}, \quad 1 \leq r \leq \infty$, for the real interpolation functors of exponent $\theta \in(0,1)$ (see [1, Section I.2], for example, for a summary of interpolation theory, and [5] or [21] for proofs), and $E_{\theta, r}:=\left(E_{0}, E_{1}\right)_{\theta, r}$ for $1 \leq r \leq \infty$ and $0<\theta<1$. Recall that

$$
E_{1} \stackrel{d}{\hookrightarrow} E_{\theta, 1} \stackrel{d}{\hookrightarrow} E_{\theta, q} \stackrel{d}{\hookrightarrow} E_{\theta, p} \hookrightarrow E_{\theta, \infty} \stackrel{d}{\hookrightarrow} E_{\vartheta, 1} \stackrel{d}{\hookrightarrow} E_{0}
$$

for $1<q<p<\infty$ and $0<\vartheta<\theta<1$. It is convenient to set $E_{j, r}:=E_{j}$ for $j=0,1$ and $1 \leq r \leq \infty$.

Let $I$ be a compact subinterval of $\mathbb{R}^{+}$containing 0 such that $\dot{I}:=I \backslash\{0\} \neq \emptyset$. If $u \in \mathbb{W}_{p}^{1}(I)$, then interpolation theory guarantees that

$$
\mathbb{W}_{p}^{1}(I):=\mathbb{W}_{p}^{1}\left(I,\left(E_{1}, E_{0}\right)\right) \hookrightarrow C\left(I, E_{1 / p^{\prime}, p}\right)
$$

(see [1, Theorem III.4.10.2]). Hence

$$
\gamma_{\tau} \in \mathcal{L}\left(\mathbb{W}_{p}^{1}(I), E_{1 / p^{\prime}, p}\right),
$$


the trace map at $t=\tau$, is well-defined by $u \mapsto u(\tau)$ for $\tau \in I$. In fact, it follows from [1, Proposition III.4.10.3] that it is a retraction. Indeed, fix any $C \in \mathcal{H}\left(E_{1}, E_{0}\right)$. Then the map $\gamma_{\tau}^{c}$, defined by

$$
\left(\gamma_{\tau}^{c} x\right)(t):=e^{-|t-\tau| C} x, \quad t \in I, \quad x \in E_{1 / p^{\prime}, p},
$$

is a coretraction for $\gamma_{\tau}$.

\section{$3 \quad$ Elementary properties}

For $T>0$ we put $J_{T}:=[0, T]$. Then we

- fix $\mathrm{T}>0$ and put $\mathrm{J}:=J_{\mathrm{\top}}$.

Henceforth, given a map $A: \mathrm{J} \rightarrow \mathcal{L}\left(E_{1}, E_{0}\right)$, we identify it with the linear map $u \mapsto A u$, defined by $(A u)(t):=A(t) u(t)$ for $t \in \mathrm{J}$ and $u: J \rightarrow E_{1}$. The relation

$$
A \in L_{1}\left(\mathrm{~J}, \mathcal{L}\left(E_{1}, E_{0}\right)\right) \cap \mathcal{L}\left(\mathbb{W}_{p}^{1}\left(\mathrm{~J},\left(E_{1}, E_{0}\right)\right), L_{p}\left(\mathrm{~J}, E_{0}\right)\right)
$$

is always to be understood in this sense.

Let (3.1) be satisfied and consider the Cauchy problem

$$
\dot{u}+A u=f \quad \text { in } \mathbf{j}, \quad u(0)=x,
$$

where

$$
(f, x) \in L_{p}\left(\mathrm{~J}, E_{0}\right) \times E_{1 / p^{\prime}, p} .
$$

By a (strong) $L_{p}\left(E_{1}\right)$ solution of $(3.2)$ (on $\mathrm{J}$ ) we mean a function $u \in \mathbb{W}_{p}^{1}(\mathrm{~J})$ satisfying (3.2) (in the point-wise sense a.e. or, equivalently, in the distributional sense). Note that, thanks to (2.5), the initial condition has a well-defined meaning.

We denote by

$$
\mathcal{M R}_{p}(\mathrm{~J}):=\mathcal{M R}_{p}\left(\mathrm{~J},\left(E_{1}, E_{0}\right)\right)
$$

the set of all $A$ satisfying (3.1) and having the property of maximal $L_{p}$ regularity on $\mathrm{J}$ with respect to $\left(E_{1}, E_{0}\right)$.

The following simple observation, for whose proof we refer to [4], is the base of our investigations.

Proposition 3.1 Let (3.1) be satisfied. Then the following are equivalent:

(i) $A \in \mathcal{M R}_{p}(\mathrm{~J})$

(ii) $\partial+A \in \mathcal{L} \operatorname{is}\left(\mathbb{W}_{p, 0}^{1}(\mathrm{~J}), L_{p}\left(\mathrm{~J}, E_{0}\right)\right)$.

(iii) $\left(\partial+A, \gamma_{0}\right) \in \mathcal{L} \operatorname{is}\left(\mathbb{W}_{p}^{1}(\mathrm{~J}), L_{p}\left(\mathrm{~J}, E_{0}\right) \times E_{1 / p^{\prime}, p}\right)$.

(iv) Problem (3.2) has for each $(f, x)$ satisfying (3.3) a unique $L_{p}\left(E_{1}\right)$ solution. 
Given $A \in \mathcal{M R}_{p}(\mathrm{~J})$, we put

$$
K:=K_{A}:=\left(\partial+A, \gamma_{0}\right)^{-1} \mid\left(L_{p}\left(\mathrm{~J}, E_{0}\right) \times\{0\}\right)
$$

and

$$
U:=U_{A}:=\left(\partial+A, \gamma_{0}\right)^{-1} \mid\left(\{0\} \times E_{1 / p^{\prime}, p}\right) .
$$

Using obvious modifications, it follows that

$$
K=(\partial+A)^{-1} \in \mathcal{L} \operatorname{is}\left(L_{p}\left(\mathrm{~J}, E_{0}\right), \mathbb{W}_{p, 0}^{1}(\mathrm{~J})\right), \quad U \in \mathcal{L}\left(E_{1 / p^{\prime}, p}, \mathbb{W}_{p}^{1}(\mathrm{~J})\right) .
$$

Clearly, $u$ is the $L_{p}\left(E_{1}\right)$ solution of (3.2) iff $u=K f+U x$.

Furthermore, for $\alpha \in \mathbb{R}^{+}$we set

$$
\mathcal{M R}_{p}(\mathrm{~J}, \alpha):=\left\{A \in \mathcal{M R}_{p}(\mathrm{~J}) ;\left\|\left(\partial+A, \gamma_{0}\right)^{-1}\right\| \leq \alpha\right\} .
$$

\section{Interval independence}

The next lemma shows that maximal regularity does not depend on the (bounded) interval. Its statement is more precise since it gives uniform bounds which are important in the sequel.

Given a map $a: \mathrm{J} \rightarrow E$ and a number $\tau \in[0, \mathrm{~T})$, we define $a_{\tau}: J_{\mathrm{T}-\tau} \rightarrow E$ by $a_{\tau}(t):=a(t+\tau)$.

Lemma 4.1 There exists $\kappa>0$ such that, given $\alpha>0$ and $A \in \mathcal{M R}_{p}(\mathrm{~J}, \alpha)$,

$$
A_{\tau} \in \mathcal{M R}_{p}\left(J_{T}, \kappa \alpha\right), \quad \tau \in[0, \mathrm{~T}), \quad T \in \dot{J}_{\mathrm{T}-\tau} .
$$

Proof. Fix $\tau \in[0, T), T \in \dot{J}_{\mathrm{T}-\tau}$, and $(g, y) \in L_{p}\left(J_{T}, E_{0}\right) \times E_{1 / p^{\prime}, p}$. Put

$$
w(t):=\gamma_{0}^{c} y(\tau-t)=e^{-(\tau-t) C} y, \quad t \leq \tau .
$$

Then $w \in \mathbb{W}_{p}^{1}(-\mathrm{T}, \tau)$ and (cf. [1, Proposition III.4.10.3]) there exists a constant $c_{0}$, independently of $\tau$ and $y$, with

$$
\|w\|_{\mathbb{W}_{p}^{1}(-\mathrm{T}, \tau)} \leq c_{0}\|y\|_{E_{1 / p^{\prime}, p}} .
$$

Hence we deduce from [1, Proposition III.1.4.2 and Lemma III.4.10.1] that

$$
x:=w(0)=e^{-\tau C} y \in E_{1 / p^{\prime}, p}
$$

and

$$
\|x\|_{E_{1 / p^{\prime}, p}} \leq c_{1}\|w\|_{\mathbb{W}_{p}^{1}(-\mathrm{T}, \tau)} \leq c_{2}\|y\|_{E_{1 / p^{\prime}, p}},
$$

where $c_{1}$ and $c_{2}:=c_{1} c_{0}$ are independent of $y$.

Set

$$
f(t):=\left\{\begin{array}{lr}
(A(t)+C) w(t), & 0 \leq t \leq \tau, \\
g(t-\tau), & \tau<t \leq \tau+T, \\
0, & \tau+T<t \leq \mathrm{T} .
\end{array}\right.
$$


Then we infer from (3.1), (4.1), and $g \in L_{p}\left(J_{T}, E_{0}\right)$ that $f \in L_{p}\left(\mathrm{~J}, E_{0}\right)$ and

$$
\|f\|_{L_{p}\left(J, E_{0}\right)} \leq c\left(\|y\|_{E_{1 / p^{\prime}, p}}+\|g\|_{L_{p}\left(J_{T}, E_{0}\right)}\right),
$$

with $c$ being independent of $\tau, y$, and $g$.

Consider the Cauchy problem

$$
\dot{v}+A(t) v=f(t), \quad t \in \dot{\mathrm{J}}, \quad v(0)=x .
$$

Then $A \in \mathcal{M R}_{p}(\mathrm{~J}, \alpha)$ implies that (4.4) has a unique solution $v \in \mathbb{W}_{p}^{1}(\mathrm{~J})$, and

$$
\|v\|_{\mathbb{W}_{p}^{1}(J)} \leq \alpha\left(\|x\|_{E_{1 / p^{\prime}, p}}+\|f\|_{L_{p}\left(J, E_{0}\right)}\right) .
$$

From this, (4.2), and (4.3) it follows that there exists $\kappa>0$, being independent of $\tau, T, y$, and $g$, such that

$$
\|v\|_{\mathbb{W}_{p}^{1}([\tau, \tau+T])} \leq \kappa \alpha\left(\|y\|_{E_{1 / p^{\prime}, p}}+\|g\|_{L_{p}\left(J_{T}, E_{0}\right)}\right) .
$$

Note that $v(t)=w(t)$ for $0 \leq t \leq \tau$ so that $v(\tau)=y$. Hence, setting $u(t):=v(t+\tau)$ for $t \in J_{T}$, we see that $u \in \mathbb{W}_{p}^{1}\left(J_{T}\right)$ and

$$
\dot{u}+A_{\tau}(t) u=g(t), \quad t \in \dot{J}_{T}, \quad u(0)=y .
$$

Moreover, from (4.5) we deduce that

$$
\|u\|_{\mathbb{W}_{p}^{1}\left(J_{T}\right)} \leq \kappa \alpha\left(\|y\|_{E_{1 / p^{\prime}, p}}+\|g\|_{L_{p}\left(J_{T}, E_{0}\right)}\right) .
$$

This shows that $A_{\tau} \in \mathcal{M R}_{p}\left(J_{T}, \kappa \alpha\right)$ and proves the lemma.

\section{Subordinate perturbations}

In this section we consider the case where an operator, possessing maximal $L_{p}$ regularity, is additively perturbed by one which has slightly better mapping properties.

Lemma 5.1 Suppose that $\alpha>0$, and $\beta: \mathrm{J} \rightarrow \mathbb{R}^{+}$is continuous at zero and satisfies $\beta(0)=0$. Then there exists $\gamma:=\gamma(\alpha, \beta)$ such that $A+B \in \mathcal{M R}_{p}(\mathrm{~J}, \gamma)$ whenever $A \in \mathcal{M R}_{p}(\mathrm{~J}, \alpha)$ and $B$ satisfies (3.1) and

$$
\left\|B_{\tau}\right\|_{\mathcal{L}\left(\mathbb{W}_{p}^{1}\left(J_{T}\right), L_{p}\left(J_{T}, E_{0}\right)\right)} \leq \beta(T), \quad \tau \in[0, T), \quad T \in \dot{J}_{T-\tau} .
$$

Proof. Suppose that $A$ and $B$ satisfy the above assumptions and $(f, x)$ fulfills (3.3). Consider the Cauchy problem

$$
\dot{u}+A(t) u+B(t) u=f(t), \quad t \in \mathbf{j}, \quad u(0)=x .
$$

It follows that (5.1) is equivalent to

$$
u+K B u=K f+U x
$$


in $\mathbb{W}_{p}^{1}(\mathrm{~J})$, and Lemma 4.1 implies

$$
\|K B\|_{\mathcal{L}\left(\mathbb{W}_{p}^{1}\left(J_{T}\right)\right)} \leq \kappa \alpha \beta(T), \quad T \in \mathbf{j} .
$$

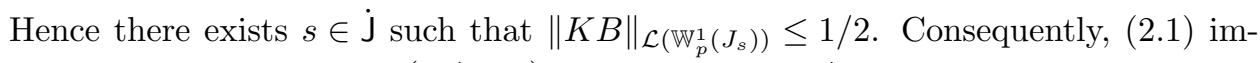
plies that $1+K B \in \mathcal{L}$ aut $\left(\mathbb{W}_{p}^{1}\left(J_{s}\right)\right)$ with $\left\|(1+K B)^{-1}\right\| \leq 2$. So we see that (5.1) has a unique $L_{p}\left(E_{1}\right)$ solution, $v_{0}$, on $J_{s}$, and

$$
\left\|v_{0}\right\|_{\mathbb{W}_{p}^{1}\left(J_{s}\right)} \leq 2 \kappa \alpha\left(\|f\|_{L_{p}\left(J_{s}, E_{0}\right)}+\|x\|_{E_{1 / p^{\prime}, p}}\right) .
$$

Put $\sigma(t):=t \wedge(\mathrm{T}-t)$ for $t \in \mathrm{J}$. Suppose that $s<\mathrm{T}$ and consider the Cauchy problem

$$
\dot{u}+A_{s}(t) u+B_{s}(t) u=f_{s}(t), \quad t \in \dot{J}_{\mathrm{T}-s}, \quad u(0)=x_{s},
$$

where $x_{s}:=v_{0}(s)$. Note that $(2.5)$ implies

$$
\left\|x_{s}\right\|_{E_{1 / p^{\prime}, p}} \leq c_{0}\left\|v_{0}\right\|_{\mathbb{W}_{p}^{1}\left(J_{s}\right)}
$$

where $c_{0}$ is the norm of the injection map (2.5) for the interval $J_{s}$. Now we infer from the preceding arguments that $(5.3)$ has a unique $L_{p}\left(E_{1}\right)$ solution, $v_{1}$, on $J_{\sigma(s)}$, and

$$
\left\|v_{1}\right\|_{\mathbb{W}_{p}^{1}\left(J_{\sigma(s)}\right)} \leq 2 \kappa \alpha\left(\left\|f_{s}\right\|_{L_{p}\left(J_{\sigma(s)}, E_{0}\right)}+\left\|x_{s}\right\|_{E_{1 / p^{\prime}, p}}\right) .
$$

Define $u_{1}$ by $u_{1}(t):=v_{0}(t)$ for $0 \leq t \leq s$, and $u_{1}(t):=v_{1}(t-s)$ for $s \leq t \leq s+\sigma(s)$. Then $u_{1}$ is an $L_{p}\left(E_{1}\right)$ solution of $(5.1)$ on $[0, s+\sigma(s)]$. Moreover, we deduce from (5.2) and (5.5) that

$$
\left\|u_{1}\right\|_{\mathbb{W}_{p}^{1}\left(J_{s+\sigma(s)}\right)} \leq c_{1}(\alpha, \beta)\left(\|f\|_{L_{p}\left(J_{s+\sigma(s)}, E_{0}\right)}+\|x\|_{\left.E_{1 / p^{\prime}, p}\right)} .\right.
$$

By repeating this argument a finite number of times, if necessary, the assertion follows.

Now it is easy to prove a first perturbation result for maximal $L_{p}$ regularity.

Theorem 5.1 Suppose that $\alpha, \beta>0$ and $p<r \leq \infty$. Then there exists $\gamma>0$ such that $A+B \in \mathcal{M R}_{p}(\mathrm{~J}, \gamma)$ whenever $A \in \mathcal{M R}_{p}(\mathrm{~J}, \alpha)$ and

$$
B \in L_{1}\left(\mathrm{~J}, \mathcal{L}\left(E_{1}, E_{0}\right)\right) \cap \mathcal{L}\left(\mathbb{W}_{p}^{1}(\mathrm{~J}), L_{r}\left(\mathrm{~J}, E_{0}\right)\right)
$$

with

$$
\|B\|_{\mathcal{L}\left(\mathbb{W}_{p}^{1}(J), L_{r}\left(J, E_{0}\right)\right)} \leq \beta .
$$

Proof. Given $u \in \mathbb{W}_{p}^{1}\left(J_{T}\right)$, put

$$
v(t):=\left\{\begin{array}{lc}
\left(\gamma_{\tau}^{c} u(0)\right)(t), & 0 \leq t \leq \tau, \\
u(t-\tau), & \tau<t<\tau+T, \\
\left(\gamma_{\tau+T}^{c} u(T)\right)(t), & \tau+T \leq t \leq \mathrm{T}
\end{array}\right.
$$


Then $v \in \mathbb{W}_{p}^{1}(\mathrm{~J})$ and

$$
\|v\|_{\mathbb{W}_{p}^{1}(J)} \leq c\|u\|_{\mathbb{W}_{p}^{1}\left(J_{T}\right)},
$$

with $c$ being independent of $u, \tau$, and $T$. Hence

$$
\begin{aligned}
\left\|B_{\tau} u\right\|_{L_{r}\left(J_{T}, E_{0}\right)} & =\|B(\tau+\cdot) u\|_{L_{r}\left(J_{T}, E_{0}\right)}=\|B v\|_{L_{r}\left((\tau, \tau+T), E_{0}\right)} \\
& \leq\|B v\|_{L_{r}\left(\mathrm{~J}, E_{0}\right)} \leq \beta\|v\|_{\mathbb{W}_{p}^{1}\left(\mathrm{~J}, E_{0}\right)} \leq c \beta\|u\|_{\mathbb{W}_{p}^{1}\left(J_{T}, E_{0}\right)} .
\end{aligned}
$$

Consequently, by Hölder's inequality,

$$
\left\|B_{\tau} u\right\|_{L_{p}\left(J_{T}, E_{0}\right)} \leq T^{\varepsilon}\left\|B_{\tau} u\right\|_{L_{r}\left(J_{T}, E_{0}\right)} \leq c \beta T^{\varepsilon}\|u\|_{\mathbb{W}_{p}^{1}\left(J_{T}, E_{0}\right)},
$$

where $\varepsilon:=1 / p-1 / r$. Hence Lemma 5.1 implies the assertion.

The following proposition gives a sufficient condition for (5.7) to be satisfied. For this we recall that

$$
\mathbb{W}_{p}^{1}\left(\mathrm{~J},\left(E_{1}, E_{0}\right)\right) \hookrightarrow\left\{\begin{array}{cl}
L_{q}\left(\mathrm{~J}, E_{\theta, 1}\right) & \text { if } 1 \geq 1 / q>\theta-1 / p^{\prime}>0, \\
C\left(\mathrm{~J}, E_{1 / p^{\prime}, p}\right) & \text { if } \theta=1 / p^{\prime} \\
C^{\rho}\left(\mathrm{J}, E_{\theta, 1}\right) & \text { if } 0 \leq \rho<1 / p^{\prime}-\theta .
\end{array}\right.
$$

Moreover, if $\theta \neq 1 / p^{\prime}$ and $E_{1}$ is compactly embedded in $E_{0}$, then these embeddings are compact as well (see [2, Theorem 3] and (2.5)).

Proposition 5.1 Suppose that $p<r$ and $0 \leq 1 / \rho<1 / r-\theta+1 / p^{\prime}$ if $\theta>1 / p^{\prime}$, and $\rho=r$ otherwise. Given $\beta_{0}>0$, there exists $\beta>0$ such that (5.7) is true for every $B$ belonging to $L_{\rho}\left(\mathrm{J}, \mathcal{L}\left(E_{\theta, \infty}, E_{0}\right)\right)$ and satisfying $\|B\| \leq \beta_{0}$.

Proof. Set $1 / q:=1 / r-1 / \rho$. Then it follows from (5.8) and (2.4) that $\mathbb{W}_{p}^{1}(\mathrm{~J})$ embeds in $L_{q}\left(\mathrm{~J}, E_{\theta, \infty}\right)$. Hence Hölder's inequality implies the validity of (5.7).

Corollary 5.1 Suppose that $\alpha, \beta>0$ and $0 \leq 1 / \rho<(1-\theta) \wedge 1 / p$. Then there exists $\gamma>0$ such that $A+B \in \mathcal{M R}_{p}(\mathrm{~J}, \gamma)$ whenever $A \in \mathcal{M R}_{p}(\mathrm{~J}, \alpha)$ and $B$ belongs to $L_{\rho}\left(\mathrm{J}, E_{\theta, \infty}\right)$ with $\|B\| \leq \beta$.

Proof. If $\theta>1 / p^{\prime}$, then fix $r$ such that $1 / p>1 / r>1 / p+1 / \rho+\theta-1$. Otherwise put $r:=\rho$. Then the assertions of Proposition 5.1 are satisfied so that Theorem 5.1 gives the assertion.

\section{The autonomous case}

We denote by $\mathcal{C}(E)$ the set of all closed linear operators in $E$. Then we set

$$
\mathcal{M R}_{p}:=\mathcal{M R}_{p}\left(E_{1}, E_{0}\right):=\mathcal{L}\left(E_{1}, E_{0}\right) \cap \mathcal{C}\left(E_{0}\right) \cap \mathcal{M R}_{p}\left(\mathrm{~J},\left(E_{1}, E_{0}\right)\right)
$$

where we identify $A \in \mathcal{L}\left(E_{1}, E_{0}\right)$ with the constant map $t \mapsto A$. Moreover, given $\alpha>0$,

$$
\mathcal{M R}_{p}[\alpha]:=\mathcal{M R}_{p}\left(E_{1}, E_{0}, \alpha\right):=\left\{A \in \mathcal{M R}_{p} ;\|A\|_{\mathcal{L}\left(E_{1}, E_{0}\right)} \leq \alpha\right\} .
$$


Remarks 6.1 (a) The assumption that $A \in \mathcal{L}\left(E_{1}, E_{0}\right) \cap \mathcal{C}\left(E_{0}\right)$ is equivalent to $A \in \mathcal{L}\left(E_{1}, E_{0}\right)$ and $E_{1} \doteq D(A)$, where $D(A)$ is the domain of $A$ (in $E_{0}$ ) endowed with its graph norm.

Proof. See Lemma I.1.1.2 in [1].

(b) $\mathcal{M R}_{p}\left(E_{1}, E_{0}\right) \subset \mathcal{H}\left(E_{1}, E_{0}\right)$.

Proof. This is a result of Dore [9] (see [1, Remark III.4.10.9(b)]).

(c) For $A \in \mathcal{H}\left(E_{1}, E_{0}\right)$ put $V(t):=e^{-t A}$. Also set

$$
V \star f(t):=\int_{0}^{t} V(t-\tau) f(\tau) d \tau, \quad f \in L_{1}\left(\mathrm{~J}, E_{0}\right), \quad t \in \mathrm{J} .
$$

Then, if $A \in \mathcal{M R}_{p}$, it follows that $K f=V \star f$ and $U x=V(\cdot) x$ for $(f, x)$ satisfying (3.3).

Proof. This follows from [1, Theorem III.1.5.2].

(d) If $A \in \mathcal{M R}_{p}\left(E_{1}, E_{0}\right)$ then $A \in \mathcal{M R}_{q}\left(E_{1}, E_{0}\right)$ for $1<q<\infty$.

Proof. This follows from (a) and [19] (also see [9]).

(e) If $A \in \mathcal{M R}_{p}\left(E_{1}, E_{0}\right)$ then $A \in \mathcal{M R}_{p}\left(J_{T},\left(E_{1}, E_{0}\right)\right)$ for every $T \in(0, \infty)$.

Proof. If $T<\mathrm{T}$, then this follows from Lemma 4.1. Otherwise we obtain the assertion by the extension argument of the last part of the proof of Lemma 5.1.

Given $\kappa \geq 1$ and $\omega>0$, we denote by $\mathcal{H}(\kappa, \omega):=\mathcal{H}\left(E_{1}, E_{0}, \kappa, \omega\right)$ the set of all $A$ in $\mathcal{L}\left(E_{1}, E_{0}\right)$ such that $\omega+A \in \mathcal{L}$ is $\left(E_{1}, E_{0}\right)$ and

$$
\kappa^{-1} \leq \frac{\|(\lambda+A) x\|_{0}}{|\lambda|\|x\|_{0}+\|x\|_{1}} \leq \kappa, \quad x \in E_{1} \backslash\{0\}, \quad \operatorname{Re} \lambda \geq \omega,
$$

where $\|\cdot\|_{j}$ is the norm in $E_{j}$. We also set

$$
\mathcal{H}(\kappa):=\mathcal{H}\left(E_{1}, E_{0}, \kappa\right):=\bigcup_{\omega>0} \mathcal{H}(\kappa, \omega) .
$$

From [1, Theorem I.2.2] we know that

$$
\mathcal{H}\left(E_{1}, E_{0}\right)=\bigcup_{\kappa \geq 1} \mathcal{H}(\kappa) .
$$

Theorem 6.1 $\mathcal{M R}_{p}\left(E_{1}, E_{0}\right)$ is open in $\mathcal{L}\left(E_{1}, E_{0}\right)$. More precisely, given $\alpha \geq 1$, it follows that $A+B \in \mathcal{M R}_{p}[2 \alpha] \cap \mathcal{H}(2 \alpha)$ whenever

$$
A \in \mathcal{M R}_{p}[\alpha] \cap \mathcal{H}(\alpha)
$$

and $B \in \mathcal{L}\left(E_{1}, E_{0}\right)$ satisfies $\|B\|_{\mathcal{L}\left(E_{1}, E_{0}\right)} \leq 1 / 2 \alpha$

Proof. First observe that Remark 6.1(b) and (6.1) imply that for each $A$ in $\mathcal{M R}_{p}$ there exists $\alpha \geq 1$ such that $A$ satisfies (6.2). Thus let $\alpha$ be fixed. Then we infer from $\|B\| \leq 1 / 2 \alpha$ and [1, Theorem I.1.3.1] that $A+B \in \mathcal{H}(2 \alpha)$. This implies that $A+B \in \mathcal{L}\left(E_{1}, E_{0}\right) \cap \mathcal{C}\left(E_{0}\right)$. 
For $(f, x) \in L_{p}\left(\mathrm{~J}, E_{0}\right) \times E_{1 / p^{\prime}, p}$ consider the Cauchy problem

$$
\dot{u}+A u+B u=f \quad \text { in } \mathbf{j}, \quad u(0)=x .
$$

Since $A \in \mathcal{M R}_{p}[\alpha],(6.3)$ is equivalent to $u+K_{A} B u=K_{A} f+U_{A} x$ in $\mathbb{W}_{p}^{1}(\mathrm{~J})$, and our assumptions imply that $\left\|K_{A} B\right\| \leq\left\|K_{A}\right\|\|B\| \leq 1 / 2$. Hence $A+B \in \mathcal{M R}_{p}[2 \alpha]$ follows from (2.1).

\section{The main theorem}

In the following basic perturbation theorem $\mathcal{M R}_{p}$ is endowed with the topology induced by $\mathcal{L}\left(E_{1}, E_{0}\right)$, of course.

Theorem 7.1 If $\vartheta, \theta \in(0,1)$ and $0 \leq 1 / \rho<(1-\theta) \wedge 1 / p$ then

$$
C\left(\mathrm{~J}, \mathcal{M R}_{p}\left(E_{1}, E_{0}\right)\right)+L_{\infty}\left(\mathrm{J}, \mathcal{L}\left(E_{1}, E_{\vartheta, \infty}\right)\right)+L_{\rho}\left(\mathrm{J}, \mathcal{L}\left(E_{\theta, \infty}, E_{0}\right)\right)
$$

is a subset of $\mathcal{M R}_{p}\left(\mathrm{~J},\left(E_{1}, E_{0}\right)\right)$.

Proof. (i) Suppose that $A \in C\left(\mathrm{~J}, \mathcal{M} \mathcal{R}_{p}\right)$. Then one verifies that

$$
\left(\tau \mapsto\left(\partial_{t}+A(\tau), \gamma_{0}\right)\right) \in C\left(\mathrm{~J}, \mathcal{L}\left(\mathbb{W}_{p}^{1}(\mathrm{~J}), L_{p}\left(\mathrm{~J}, E_{0}\right) \times E_{1 / p^{\prime}, p}\right)\right) .
$$

Since $\left(\partial_{t}+A(\tau), \gamma_{0}\right) \in \mathcal{L}$ is $\left(\mathbb{W}_{p}^{1}(\mathrm{~J}), L_{p}\left(\mathrm{~J}, E_{0}\right) \times E_{1 / p^{\prime}, p}\right)$ for each $\tau \in \mathrm{J}$, it follows from the smoothness of the inversion map (2.2) that

$$
\left(\tau \mapsto\left(\partial_{t}+A(\tau), \gamma_{0}\right)^{-1}\right) \in C\left(\mathrm{~J}, \mathcal{L}\left(L_{p}\left(\mathrm{~J}, E_{0}\right) \times E_{1 / p^{\prime}, p}, \mathbb{W}_{p}^{1}(\mathrm{~J})\right)\right) .
$$

Hence, by the compactness of $\mathrm{J}$, there exists $\alpha>0$ such that $A(\tau) \in \mathcal{M R}_{p}[\alpha]$ for $\tau \in J$. Thus we deduce from Lemma 4.1 that there exists $\kappa>0$ such that

$$
(t \mapsto A(\tau)) \in \mathcal{M R}_{p}\left(J_{T}, \kappa \alpha\right), \quad \tau \in[0, \mathrm{~T}), \quad T \in \dot{J}_{\mathrm{T}-\tau} .
$$

(ii) Since $A \in C\left(\mathrm{~J}, \mathcal{M R}_{p}\left(E_{1}, E_{0}\right)\right)$ we see from Remark 6.1(b) that $A$ belongs to $C\left(\mathrm{~J}, \mathcal{H}\left(E_{1}, E_{0}\right)\right)$. Hence the compactness of $A(\mathrm{~J})$ and [1, Corollary I.1.3.2] imply that there exist $\omega>0$ and $\kappa \geq 1$ with

$$
A(\tau) \in \mathcal{H}\left(E_{1}, E_{0}, \kappa, \omega\right), \quad \tau \in \mathrm{J} .
$$

From this and [1, Corollary I.1.4.3 and Lemma II.4.2.1] it follows that there is a constant $c_{0}$ such that

$$
\left\|e^{-t A(\tau)}\right\|_{\mathcal{L}\left(E_{j}\right)}+t\left\|e^{-t A(\tau)}\right\|_{\mathcal{L}\left(E_{0}, E_{1}\right)} \leq c_{0}, \quad t \in \mathbf{j}, \quad \tau \in \mathrm{J} .
$$

Hence [2, Lemma 3(i)] and Remark 6.1(c) guarantee the existence of a constant $c_{0}$ satisfying

$$
\left\|K_{A(\tau)} u\right\|_{L_{p}\left(J_{T}, E_{1}\right)} \leq c_{0} T^{\vartheta}\|u\|_{L_{p}\left(J_{T}, E_{\vartheta, \infty}\right)}, \quad T \in[0, T), \quad \tau \in \mathrm{J},
$$


for $u \in L_{p}\left(J_{T}, E_{\vartheta, \infty}\right)$.

(iii) Suppose that $B \in L_{\infty}\left(\mathrm{J}, \mathcal{L}\left(E_{1}, E_{\vartheta, \infty}\right)\right)$ and that the norm of this map is bounded by $\beta$. Then

$$
\left\|B_{\tau}\right\|_{L_{\infty}\left(J_{T}, \mathcal{L}\left(E_{1}, E_{\vartheta}, \infty\right)\right)} \leq \beta, \quad \tau \in[0, \mathrm{~T}), \quad T \in J_{\mathrm{T}-\tau} .
$$

Thus (7.2) implies

$$
\left\|K_{A(\tau)} B_{\tau}\right\|_{\mathcal{L}\left(L_{p}\left(J_{T}, E_{1}\right)\right)} \leq c_{0} \beta T^{\vartheta}, \quad \tau \in[0, T), \quad T \in J_{\mathrm{T}-\tau} .
$$

Note that (7.1) and Lemma 4.1 guarantee the existence of a constant $c_{1}$ for which

$$
\left\|K_{A(\tau)}\right\|_{\mathcal{L}\left(L_{p}\left(J_{T}, E_{0}\right), \mathbb{W}_{p}^{1}\left(J_{T}, E_{1}\right)\right)} \leq c_{1}, \quad \tau \in[0, \mathrm{~T}), \quad T \in \dot{J}_{\mathrm{T}-\tau} .
$$

From this, $E_{\vartheta, \infty} \hookrightarrow E_{0},(7.3)$, and (7.4) we infer

$$
\left\|B_{\tau}\left(K_{A(\tau)} B_{\tau}\right)^{j} K_{A(\tau)}\right\|_{\mathcal{L}\left(L_{p}\left(J_{T}, E_{0}\right)\right)} \leq c_{1} \beta\left(c_{0} \beta T^{\vartheta}\right)^{j}, \quad j \in \mathbb{N}^{\times},
$$

for $\tau \in[0, \mathrm{~T})$ and $T \in \dot{J}_{\mathrm{T}-\tau}$. Consequently, there exists $T_{0} \in \mathbf{j}$ such that

$$
R_{\tau}:=1-B_{\tau} K_{A(\tau)}+\sum_{j=1}^{\infty}(-1)^{j-1} B_{\tau}\left(K_{A(\tau)} B_{\tau}\right)^{j} K_{A(\tau)}
$$

is a well-defined element in $\mathcal{L}\left(L_{p}\left(J_{T}, E_{0}\right)\right)$ for $T \in \dot{J}_{T_{0}} \cap J_{\boldsymbol{\top}-\tau}$, and there exists $\kappa_{1}$ with

$$
\left\|R_{\tau}\right\|_{\mathcal{L}\left(L_{p}\left(J_{T}, E_{0}\right)\right)} \leq \kappa_{1}, \quad T \in \dot{J}_{T_{1}} \cap \dot{J}_{\mathrm{T}-\tau}, \quad \tau \in[0, \mathrm{~T}) .
$$

It is easily verified that $R_{\tau}=\left(1+B_{\tau} K_{A(\tau)}\right)^{-1}$.

(iv) By the uniform continuity of $A$ there exists $\varepsilon>0$ such that

$$
\|A(s)-A(t)\|_{\mathcal{L}\left(E_{1}, E_{0}\right)} \leq 1 / 2 c_{1} \kappa_{1}, \quad s, t \in \mathrm{J}, \quad|s-t| \leq \varepsilon .
$$

Fix $T \in(0, \varepsilon]$ and $m \in \mathbb{N}$ such that $m T=\mathrm{T}$. Put $\tau_{j}:=j T$ and

$$
C_{j}(t):=A\left(t+\tau_{j}\right)-A\left(\tau_{j}\right) .
$$

Then, given $f \in L_{p}\left(J_{T}, E_{0}\right)$, the Cauchy problem

$$
\dot{u}+(A+B)_{\tau_{j}}(t) u=f(t), \quad t \in \dot{J}_{T}, \quad u(0)=0
$$

can be rewritten as

$$
\dot{u}+A\left(\tau_{j}\right) u+B_{\tau_{j}}(t) u=f(t)-C_{j}(t) u, \quad t \in \dot{J}_{T}, \quad u(0)=0 .
$$

Since $\partial+A\left(\tau_{j}\right)+B_{\tau_{j}}=\left(1+B_{\tau_{j}} K_{A\left(\tau_{j}\right)}\right)\left(\partial_{t}+A\left(\tau_{j}\right)\right)$ we see that (7.8) is equivalent to

$$
\left(1+K_{A\left(\tau_{j}\right)} R_{\tau_{j}} C_{j}\right) u=K_{A\left(\tau_{j}\right)} R_{\tau_{j}} f
$$


in $\mathbb{W}_{p}^{1}\left(J_{T}\right)$. Note that $(7.5)-(7.7)$ imply

$$
\left\|K_{A\left(\tau_{j}\right)} R_{\tau_{j}} C_{j}\right\|_{\mathcal{L}\left(\mathbb{W}_{p}^{1}\left(J_{T}\right)\right)} \leq 1 / 2, \quad 0 \leq j \leq m .
$$

Thus (7.9) has a unique solution $u_{j} \in \mathbb{W}_{p}^{1}\left(J_{T}\right)$ and

$$
\left\|u_{j}\right\|_{\mathbb{W}_{p}^{1}\left(J_{T}\right)} \leq 2 c_{1} \kappa_{1}\|f\|_{L_{p}\left(J_{T}, E_{0}\right)}, \quad 0 \leq j \leq m .
$$

From (7.1) we also infer that

$$
\left\|U_{A\left(\tau_{j}\right)}\right\|_{\mathcal{L}\left(E_{1 / p^{\prime}, p}, \mathbb{W}_{p}^{1}(J)\right)} \leq \kappa \alpha, \quad 0 \leq j \leq m .
$$

Thus, given $j \in\{0, \ldots, m\}, \quad u_{j}+U_{A\left(\tau_{j}\right)} x \in \mathbb{W}_{p}^{1}(J)$ is, for each $(f, x)$ belonging to $L_{p}\left(J_{T}, E_{0}\right) \times E_{1 / p^{\prime}, p}$, the unique solution of the Cauchy problem

$$
\dot{u}+(A+B)_{\tau_{j}}(t) u=f(t), \quad t \in \dot{J}_{T}, \quad u(0)=x .
$$

Now the continuation argument of the last part of the proof of Lemma 5.1 implies that $A+B$ belongs to $\mathcal{M R}_{p}\left(\mathrm{~J},\left(E_{1}, E_{0}\right)\right)$ whenever $A \in C\left(\mathrm{~J}, \mathcal{M R}_{p}\left(E_{1}, E_{0}\right)\right)$ and $B \in L_{\infty}\left(\mathrm{J}, \mathcal{L}\left(E_{1}, E_{\vartheta, \infty}\right)\right)$.

(v) Lastly, the assertion follows from what has just been shown and Corollary 5.1.

Corollary 7.1 If $\vartheta, \theta \in(0,1)$ then

$$
\mathcal{M R}_{p}\left(E_{1}, E_{0}\right)+\mathcal{L}\left(E_{1}, E_{\vartheta, \infty}\right)+\mathcal{L}\left(E_{\theta, \infty}, E_{0}\right) \subset \mathcal{M R}_{p}\left(E_{1}, E_{0}\right) .
$$

The following proposition shows that, given a continuous map $A$, it is not more general to assume that $A$ has maximal regularity than to presuppose that this is true for every constant operator $A(\tau)$.

Proposition 7.1 If $A \in \mathcal{M R}_{p}\left(\mathrm{~J},\left(E_{1}, E_{0}\right)\right) \cap C\left(\mathrm{~J}, \mathcal{L}\left(E_{1}, E_{0}\right)\right)$ then $A(\tau)$ belongs to $\mathcal{M R}_{p}\left(E_{1}, E_{0}\right)$ for $0 \leq \tau<\mathrm{T}$.

Proof. Fix $\alpha>0$ such that $A \in \mathcal{M R}_{p}(\mathrm{~J}, \alpha)$. Also fix $\tau \in[0, \mathrm{~T}]$ and $T \in \dot{J}_{\mathrm{T}-\tau}$ such that

$$
\|A(t)-A(\tau)\|_{\mathcal{L}\left(E_{1}, E_{0}\right)} \leq 1 / 2 \alpha, \quad \tau \leq t \leq \tau+T .
$$

Then

$$
\left\|\left(A(\tau)-A_{\tau}\right) K_{A_{\tau}}\right\|_{L_{p}\left(J_{T}, E_{0}\right)} \leq 1 / 2
$$

and it follows from

$$
\partial_{t}+A(\tau)=\left(1+\left(A(\tau)-A_{\tau}\right) K_{A_{\tau}}\right)\left(\partial_{t}+A_{\tau}\right)
$$

(2.1), and Lemma 4.1 that

$$
\partial_{t}+A(\tau) \in \mathcal{L} \operatorname{is}\left(\mathbb{W}_{p}^{1}\left(J_{T},\left(E_{1}, E_{0}\right)\right), L_{p}\left(J_{T}, E_{0}\right)\right) .
$$

Hence $A(\tau)$ has the property of maximal $L_{p}$ regularity with respect to the interval $J_{T}$. Now the assertion follows from Remark 6.1(e). 
Remarks 7.1 (a) In [17, Theorem 2.5] it is shown that each

$$
A \in C\left(\mathrm{~J}, \mathcal{H}\left(E_{1}, E_{0}\right)\right) \text { satisfying } A(t) \in \mathcal{M R}_{p}\left(E_{1}, E_{0}\right)
$$

has maximal $L_{p}$ regularity by constructing an evolution operator and by a rather complicated proof. Because of Remark 6.1(b) this is a special case of Theorem 7.1.

The second main result of [17], namely Theorem 3.1 therein, says the following: Let $A$ satisfy (7.10) and suppose that $B(t) \in \mathcal{L}\left(E_{1 / q^{\prime}, q}, E_{0}\right)$ for some $q \in[1, \infty)$, that $B(\cdot) x$ is measurable for each $x \in E_{1 / q^{\prime}, q}$, and that $\|B(t)\|_{\mathcal{L}\left(E_{1 / q^{\prime}, q}, E_{0}\right)} \leq \beta(t)$ for a.a. $t \in \mathrm{J}$ and some $\beta \in L_{\rho}(\mathrm{J})$, where $q<\rho \leq \infty$. Then $A+B \in \mathcal{M R}_{p}\left(\mathrm{~J},\left(E_{1}, E_{0}\right)\right)$, provided $p<\rho / q$.

This assumption implies

$$
B \in L_{\rho}\left(\mathrm{J}, \mathcal{L}\left(E_{1 / q^{\prime}, q}, E_{0}\right)\right)
$$

(see [14, Theorems 3.5.3 and 3.5.5]). Furthermore, since the inequalities involving $q$ and $\rho$ are strict, by increasing $q$ slightly we can, because of (2.4), replace $E_{1 / q^{\prime}, q}$ by $E_{1 / q^{\prime}, \infty}$. Then, setting $\theta:=1 / q^{\prime}$, the perturbation theorem of Prüss and Schnaubelt guarantees that $A+B$ has maximal $L_{p}$ regularity if $0 \leq 1 / \rho<(1-\theta) / p$. This condition is stronger than our hypothesis $0 \leq 1 / \rho<(1-\theta) \wedge 1 / p$. Thus their perturbation theorem is a particular case of Theorem 7.1, whose proof is much easier than the one for Theorem 3.1 in [17].

It should be noted that Proposition 7.1 essentially coincides with Remark 2.6 of [17], except that there it is already assumed that $A(\tau) \in \mathcal{H}\left(E_{1}, E_{0}\right)$ for $\tau \in \mathrm{J}$.

(b) For maximal regularity results for nonautonomous equations with not necessarily constant domains we refer to [12], [13], and to [20], where the latter author builds on $\mathcal{R}$-boundedness results, however. In this connection we also mention [16], where an analogue to Theorem 6.1 for $\mathcal{R}$-boundedness is proved.

\section{References}

[1] H. Amann. Linear and quasilinear parabolic problems. Vol. I, volume 89 of Monographs in Mathematics. Birkhäuser Boston Inc., Boston, MA, 1995. Abstract linear theory.

[2] H. Amann. Linear parabolic problems involving measures. Rev. R. Acad. Cien. Serie A. Mat. (RACSAM), 95 (2001), 85-119.

[3] H. Amann. Linear parabolic equations with singular potentials. J. Evol. Equ., 3 (2003), 395-406.

[4] H. Amann. Nonautonomous parabolic equations involving measures. Zapiski Nauchn. Seminar. POMI, 306 (2003), 16-52.

[5] J. Bergh and J. Löfström. Interpolation Spaces. An Introduction. Springer Verlag, Berlin, 1976. 
[6] J. Bourgain. Vector valued singular integrals and the $H^{1}$-BMO duality. In Israel seminar on geometrical aspects of functional analysis (1983/84), pages XVI, 23. Tel Aviv Univ., Tel Aviv, 1984.

[7] Ph. Clément and J. Prüss. An operator-valued transference principle and maximal regularity on vector-valued $L_{p}$-spaces. In Evolution equations and their applications in physical and life sciences (Bad Herrenalb, 1998), volume 215 of Lecture Notes in Pure and Appl. Math., pages 67-87. Dekker, New York, 2001.

[8] R. Denk, M. Hieber and J. Prüss. R-boundedness, Fourier multipliers and problems of elliptic and parabolic type. Memoires Amer. Math. Soc., (2003). To appear.

[9] G. Dore. $L^{p}$ regularity for abstract differential equations. In H. Komatsu, editor, Functional Analysis and Related Topics, 1991, pages 25-38, Berlin, 1993. Proceedings Kyoto 1991, Lecture Notes in Math. \#1540, Springer Verlag.

[10] G. Dore and A. Venni. On the closedness of the sum of two closed operators. Math. Z., 196 (1987), 189-201.

[11] J. Escher, J. Prüss, and G. Simonett. Analytic solutions for the Stefan problem with Gibbs-Thomson correction. J. reine angew. Math. To appear.

[12] M. Hieber and S. Monniaux. Heat-kernels and maximal $L^{p}-L^{q}$-estimates: the non-autonomous case. J. Fourier Anal. Appl., 6(5) (2000), 467-481.

[13] M. Hieber and S. Monniaux. Pseudo-differential operators and maximal regularity results for non-autonomous parabolic equations. Proc. Amer. Math. Soc., 128(4) (2000), 1047-1053.

[14] E. Hille and R.S. Phillips. Functional Analysis and Semi-Groups. Amer. Math. Soc., Providence, R.I., 1957.

[15] N. J. Kalton and G. Lancien. A solution to the problem of $L^{p}$-maximal regularity. Math. Z., 235(3) (2000), 559-568.

[16] P.Ch. Kunstmann and L. Weis. Perturbation theorems for maximal $L_{p^{-}}$ regularity. Ann. Scuola Norm. Sup. Pisa Cl. Sci. (4), 30(2) (2001), 415-435.

[17] J. Prüss and R. Schnaubelt. Solvability and maximal regularity of parabolic evolution equations with coefficients continuous in time. J. Math. Anal. Appl., 256(2) (2001), 405-430.

[18] L. De Simon. Un'applicazione della teoria degli integrali singolari allo studio delle equazioni differenziali astratte del primo ordine. Rend. Sem. Mat. Univ. Padova, 34 (1964), 205-232. 
[19] P.E. Sobolevskii. Coerciveness inequalities for abstract parabolic equations. Soviet Math. (Doklady), 5 (1964), 894-897.

[20] Ž. Štrkalj. R-Beschränktheit, Summensätze abgeschlossener Operatoren und operatorwertige Pseudodifferentialoperatoren. PhD thesis, Karlsruhe, 2000.

[21] H. Triebel. Interpolation Theory, Function Spaces, Differential Operators. North Holland, Amsterdam, 1978.

[22] L. Weis. A new approach to maximal $L_{p}$-regularity. In Evolution equations and their applications in physical and life sciences (Bad Herrenalb, 1998), volume 215 of Lecture Notes in Pure and Appl. Math., pages 195-214. Dekker, New York, 2001.

[23] L. Weis. Operator-valued Fourier multiplier theorems and maximal $L_{p^{-}}$ regularity. Math. Ann., 319(4) (2001), 735-758. 